This material is published in the open archive of Mid Sweden University

DIVA http://miun.diva-portal.org to ensure timely dissemination of scholarly and technical work. Copyright and all rights therein are retained by authors or by other copyright holders. All persons copying this information are expected to adhere to the terms and constraints invoked by each author's copyright. In most cases, these works may not be reposted without the explicit permission of the copyright holder.

Damghanian M.; Olsson, R; Sjöström, M., "Depth and Angular Resolution in Plenoptic Cameras," in 2015 IEEE International Conference on Image Processing (ICIP), September 27-30 2015

(C) 2015 IEEE. Personal use of this material is permitted. Permission from IEEE must be obtained for all other uses, in any current or future media, including reprinting/republishing this material for advertising or promotional purposes, creating new collective works, for resale or redistribution to servers or lists, or reuse of any copyrighted component of this work in other works." 


\title{
DEPTH AND ANGULAR RESOLUTION IN PLENOPTIC CAMERAS
}

\author{
M. Damghanian, R. Olsson, M. Sjöström \\ Department of Information and Communication Systems, \\ Mid Sweden University, Sundsvall, Sweden
}

\begin{abstract}
We present a model-based approach to extract the depth and angular resolution in a plenoptic camera. Obtained results for the depth and angular resolution are validated against Zemax ray tracing results. The provided model-based approach gives the location and number of the resolvable depth planes in a plenoptic camera as well as the angular resolution with regards to disparity in pixels. The provided model-based approach is straightforward compared to practical measurements and can reflect on the plenoptic camera parameters such as the microlens f-number in contrast with the principalray-model approach. Easy and accurate quantification of different resolution terms forms the basis for designing the capturing setup and choosing a reasonable system configuration for plenoptic cameras. Results from this work will accelerate customization of the plenoptic cameras for particular applications without the need for expensive measurements.
\end{abstract}

Index Terms - Plenoptic capture, SPC model, angular resolution, depth resolution, camera evaluation

\section{INTRODUCTION}

A plenoptic camera is built by adding a microlens array to a conventional camera, between the main lens and the image sensor and closer to the image sensor $[1,2]$. The resulting camera structure records both spatial and angular information about the scene and so opens up for a range of applications such as single snapshot 3D, refocusing and extended depth of field, naming a few $[3,4,5]$. The gain for angular information is obtained at the cost of the sensor pixel budget and postcapture computations. The two configurations of the plenoptic cameras, introduced in [2] and [6] respectively, provide different spatio-angular tradeoff of resolution. It is important to quantify the resolution terms for camera adjustment and evaluation, as well as application-based customization purposes.

This work has been supported by grant 20120328 of the KK Foundation, Sweden, and by grant 00174636 of the EU European Regional Development Fund, Mellersta Norrland, Sweden.

\subsection{Prior Work}

Performance of a plenoptic camera can be expressed in a number of high level camera parameters such as spatial and angular resolution on different depth planes, number and location of resolvable depth planes, depth of field, etc. Analytical and model based approaches have been utilized for quantifying the performance of a plenoptic camera such as the efforts in $[6,7,8]$. An analytical approach for calculating the spatial resolution in a multi-focus plenoptic camera is also presented in [9]. The analytical approaches provide a closed form formula and are the simplest to use, though they employ simplifying assumptions and so do not have the desired level of details and accuracy and can hardly be generalized. Experimental approaches are located in the other end of the complexity spectrum for evaluating plenoptic cameras. Examples of such approaches can be found in [8, 10, 11]. Experimental approaches are too expensive since they need a controlled environment, proper equipments and a minimum level of expertise. Any minor adjustments in camera parameters such as changing the focus or aperture size asks for a whole new set of measurements. Consequently, experimental measurements are mostly used for validation of the analytical or model based approaches. To conclude, a straightforward and accurate solution for performance evaluation of a plenoptic camera is of interest and model-based approaches can be the promising alternative.

\subsection{SPC model for camera evaluations}

The Sampling Pattern Cube (SPC) model was introduced in [12] and has been successfully utilized for extraction of the spatial resolution in integral imaging setups [13] and state of the art plenoptic cameras $[14,15]$. In contrast to the previous models, the SPC model considers the light samples in the form of light cones, which are bundles of light rays, with a tip position and an angular span (see Figure 1). Each pixel on the image sensor receives a bundle of light rays in the form of a light cone and the recorded value by that pixel is the sum of the irradiance of all the light rays in that light cone. To investigate the light sampling properties of the camera, first the SPC model of the camera is built. For this purpose, the light cones are back-tracked to the object space outside the camera. The result of the back-tracking process, which is a new set of light 


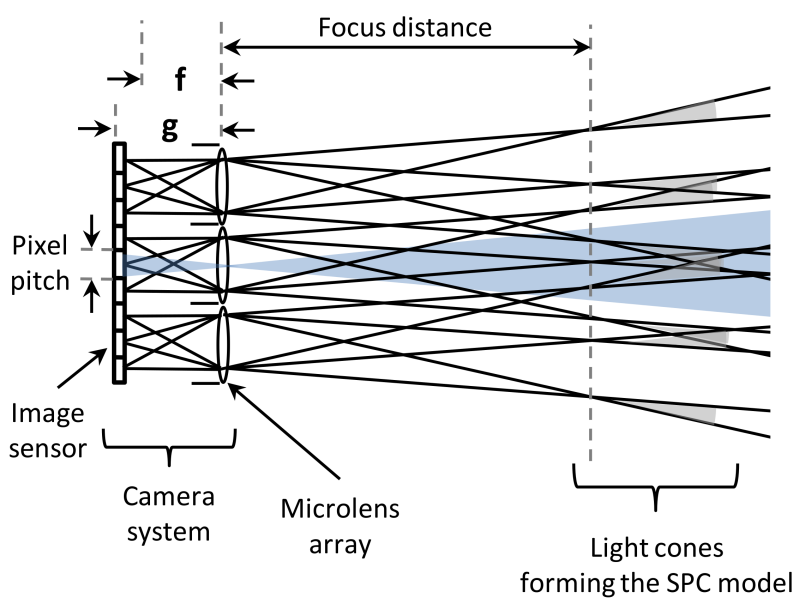

Fig. 1: The SPC model in a plenoptic capturing setup consisting of an image sensor and a microlens array.

cones this time in the abject space, is termed the SPC model of the capturing system (see Figure 1). The focus properties of the plenoptic camera is represented in the angular span of the light cones. The camera properties such as the spatial resolution in various depth planes is extracted by looking into the distribution and the overlaps of the light cones in the SPC model of the camera. In the process of extracting the spatial resolution of plenoptic cameras, the SPC model has offered lower complexity compared to the wave-optic-based models and higher accuracy relative to the principal-ray-model which assigns a single principal ray to each pixel [13]. The SPC model is bound to the realm of the geometrical optics which gives a wider scope compared to e.g. the paraxial ray model utilized in [6, 8]. Considering the above advantages, this work focuses on quantifying the angular and depth resolution in a plenoptic camera using the SPC model. Section 2 defines angular and depth resolution extractors and describes their implementation. Results and discussion will follow in Section 3 including validation of the SPC-model-based depth and angular resolution extractors against optical simulation results. Finally we conclude the work in Section 4.

\section{DEPTH AND ANGULAR RESOLUTION EXTRACTOR}

The depth resolution and the angular resolution are closely related. The depth resolution is looking into the spacing between two consecutive resolvable depth planes while the angular resolution looks into the required disparity amount (in pixels or in angle units) in order to get a new view. The two terms are illustrated in Figure 2.

Definition 1 (Depth resolvability in the SPC model). Two depth planes $z_{1}$ and $z_{2}$ are defined resolvable if not exactly

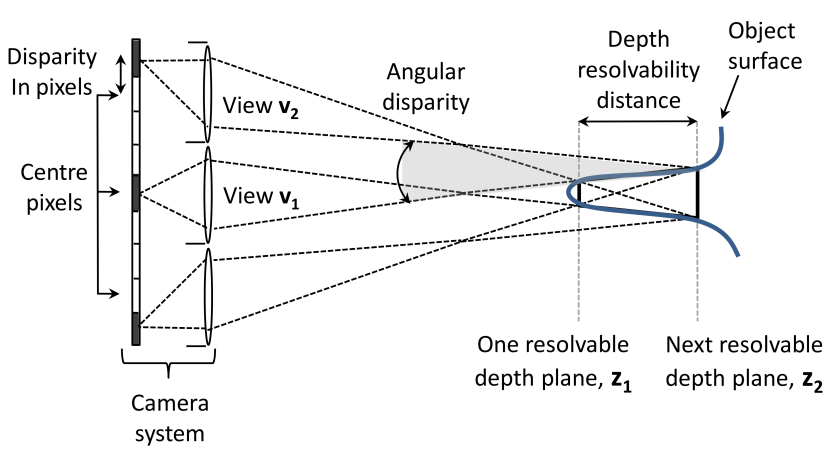

Fig. 2: Illustrating the depth resolution and the angular resolution in a plenoptic capturing setup.

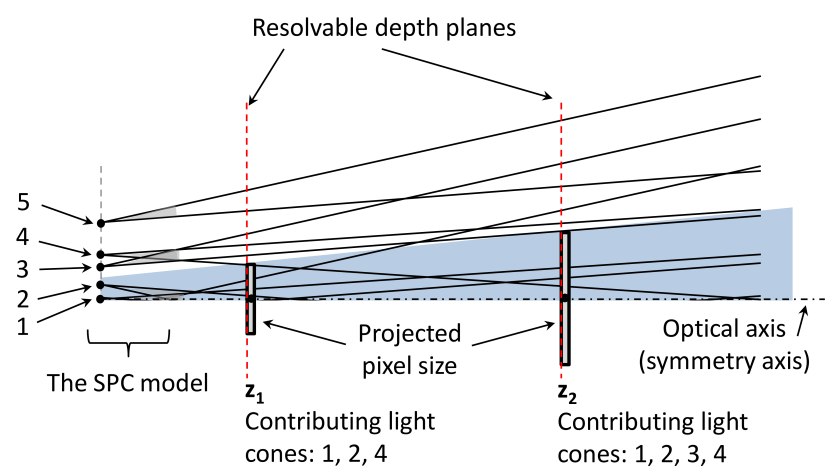

Fig. 3: Depth resolution in a focused plenoptic camera. Because of axial symmetry we show only half of the capturing system. A number of light cones (naming 1,2 and 4) contribute to the pixel value in the depth plane of interest $z_{1}$. Moving towards depth plane $z_{2}$, the change in the list of the contributing light cones (addition of light cone 3 ) makes the two depth planes $z_{1}$ and $z_{2}$ resolvable.

same list of light cones contribute to the corresponding pixel values on those two depth planes.

Using Definition 1, we build the SPC-model-based depth resolution extractor by:

1. Drawing the line showing the boundary of the central projected pixel (shaded area in Figures 1 and 3). The projected pixel size grows proportionally with depth.

2. Moving in depth towards farther depth planes and marking the depth planes where a new light cone intersects the boundary of the central projected pixel, as the next resolvable depth plane.

Figure 3 illustrates how the resolvable depth planes are extracted, using the SPC model of the plenoptic capturing setup previously shown in Figure 1. The depth-resolution extractor searches in depth and marks the depth planes (in this case depth planes $z_{1}$ and $z_{2}$ ), where a new light cone enters the 


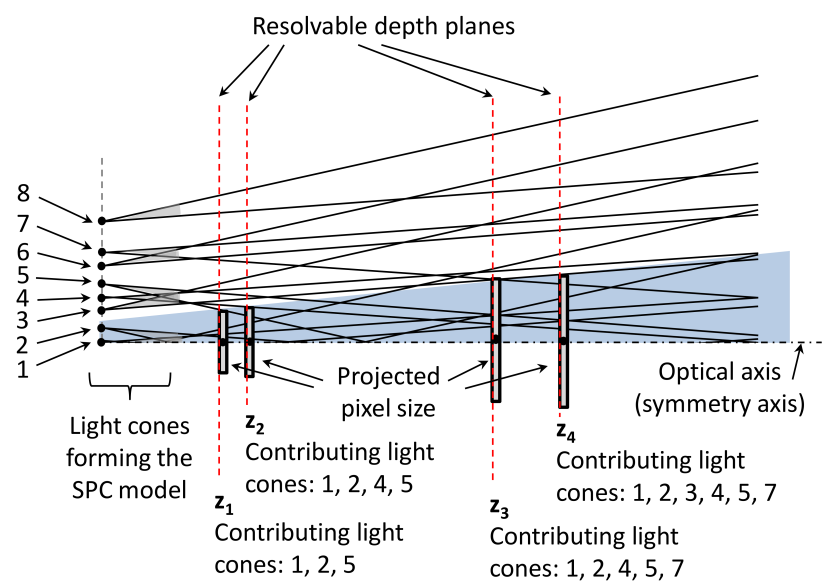

Fig. 4: Increasing the number of microlenses (compared to the case in Figure 3) provides new angular information and so improves the depth resolution. The plenoptic setup in this figure is similar to the setup shown in Figure 1, except having five microlenses and so 15 pixels in total in a row. Only half of the SPC model, and so only eight light cones, are illustrated because of the axial symmetry.

list of contributing light cones to the pixel value. The depth planes $z_{i}, i=1, \ldots, n$, which are marked by the depth resolution extractor, are the location of the resolvable depth planes for the investigated plenoptic camera. For illustration purposes, the main lens is discarded in Figures 1 to 4 . However, it is straightforward to transform the location of depth planes to the object space using the thin lens equation:

$$
\frac{1}{a}+\frac{1}{b}=\frac{1}{F}
$$

where $a$ and $b$ are object and image distances to the main lens and $F$ is the focal length.

The angular resolution is obtained with a method similar to the resolvable depth planes $z_{i}$. We quantify the angular resolution with regards to the disparity in pixels. To extract the angular resolution, first we assume that the disparity between two neighbouring microimages is considered. We build the SPC-model-based angular resolution extractor by:

1. Building the SPC model of the capturing system using only the two neighbouring microlenses.

2. Finding the resolvable depth planes corresponding to the contribution of the light cones belonging to the neighbouring microlens. An example would be the depth plane where the light cone 5 intersects the boundary of the central projected pixel in Figure 3 (This depth plane happens farther in depth and so is not shown explicitly in Figure 3).

3. Looking up the image sensor pixel corresponding to the light cone, for each light cone from the neighbouring

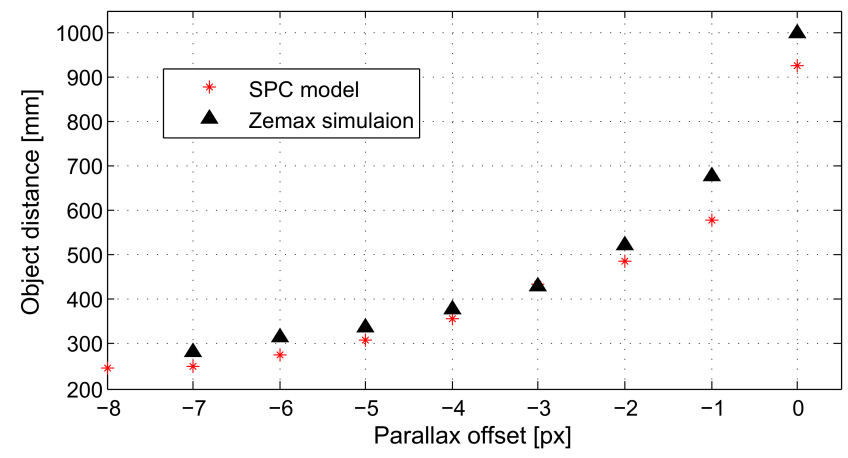

Fig. 5: Parallax offset results from the SPC model-based extractor and Zemax simulations. Both depth and angular resolution extractors are utilized to get the SPC model-based parallax offset results.

microlens that marked a resolvable depth plane. E.g. the light cone 5 in Figure 3 corresponds to the lower pixel in the upper most microimage in Figure 1. This is done using the correspondence list (the list that preserves the correspondence between light cones and image pixels) in the SPC model.

4. Counting the number of disparity pixels (with respect to the center of the microlens). For the light cone 5 , the disparity is -1 pixel.

If the disparity between two non neighboring microimage is of interest, the SPC model of the capturing system is built using only those two microlenses. The rest of the procedure will be as before. The depth and angular resolution extractors are implemented in Matlab. The source code for the SPC model implementation is accessible at http://github. $\mathrm{com} / \mathrm{mitigooli/OpenAccessSPC}$.

\section{RESULT AND DISCUSSION}

We validate the SPC-based depth and angular resolution extractors results against the Zemax simulation results from [8]. For this purpose, we build the SPC model of the plenoptic camera configuration stated in [8], and apply the defined depth and resolution extractors. Figure 5 illustrates resolvable depth planes for every one pixel parallax in the microimages captured by the neighbouring microlenses. In order to obtain the results in Figure 5, the depth resolution extractor has marked the resolvable depth planes and then the angular resolution extractor has translated them to the parallax offset in pixels. The SPC-model-based results in Figure 5 are well in line with the results from Zemax ray tracing (from [8]), though the SPC-model based approach is much more straightforward since it does not require a full-fledged ray tracing simulation. As illustrated in Figure 5, the resolvable depth planes are not distributed uniformly throughout depth. 


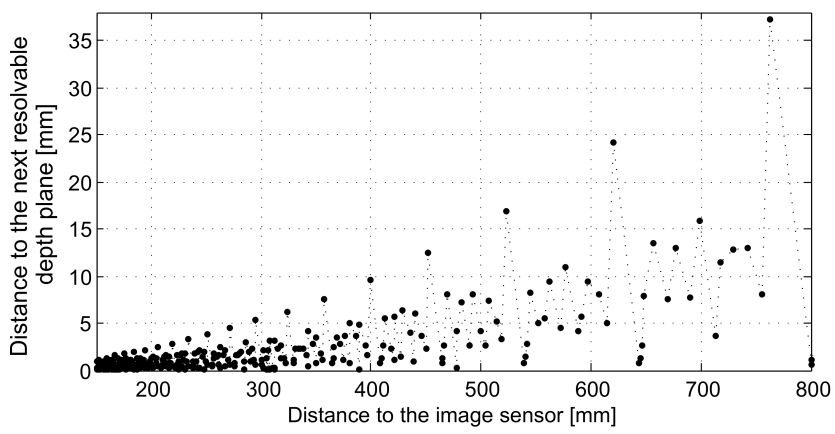

Fig. 6: The distance between consecutive resolvable depth planes in a plenoptic setup. Results are obtained by applying the SPC-based depth resolution extractor to the integral imaging setup specified in [13].

Figure 4 gives an insight into this observation. Contribution of new light cones, which gives the position of the resolvable depth planes, does not occur uniformly in depth and the transformation through the main lens only amplifies this nonuniformity. Figure 6 illustrates the distance between consecutive resolvable depth planes in an integral imaging setup specified in [13], using the SPC-based depth resolution extractor. Figure 6 shows that by going farther from the camera, the period and amplitude of the cycles increase (but not necessarily the distance between two consecutive resolvable depth planes). One reasoning for the existence of such periodicity is the periodic structure of the microlens array. Comparing Figures 3 and 4, increasing the number of image sensor pixels and the microlenses (without shrinking the pixel size or microlens pitch) will increase the number and refine the previous distribution of the resolvable depth planes.

In practice, tolerances in the microlens fabrication process and assembling the plenoptic camera might cause deviations from the design parameters. For adjustment and evaluation purposes, it is of great importance to quantify the extent of the deviations. One example is the fabrication tolerance of the microlens aperture size. Figure 7 illustrates the effect of the microlens f-number variation on the parallax offset, using the SPC model-based angular resolution extractor. Here the variation of the fabricated aperture opening from the designed value for all microlenses is of interest, not the variation between the aperture size of different microlenses in the array. Other approaches based on the principal ray model (such as [11]) can not quantify the effect of varying f-number since they do not consider the variations in the span of the light samples as they get farther from the aperture. However, the ray tracing ([8]) approaches can quantify the effect of varying f-number, but only with a full-fledged simulation. The SPC model performs the task using only the camera geometry.

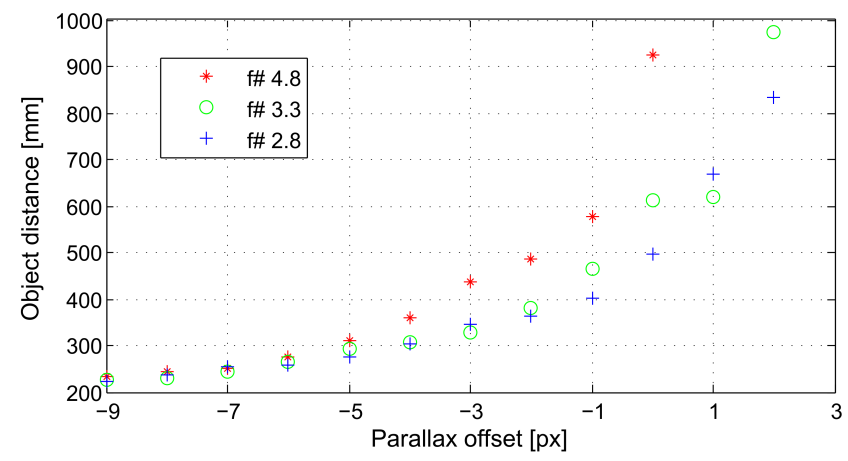

Fig. 7: Illustrating the effect of the microlens f-number variation on the parallax offset.

\section{CONCLUSION}

In this work we provided depth and angular resolution extractors for plenoptic cameras using the SPC model. We have validated the SPC-based extractors for the depth and angular resolution against Zemax ray tracing results and successfully quantified the number and location of the resolvable depth planes in a plenoptic camera. We showed that the provided extractors reflect how the variations in the plenoptic setup such as the number of pixels and microlenses and the f-number of the microlenses affect the number and location of resolvable depth planes. The provided extractors are shown to be more accurate compared to the principal-ray model while do not require a full-fledged simulation contrary to the comparable ray tracing approaches. This work confirms suitability of the SPC model for the performance evaluation of the plenoptic cameras. Together with a previously defined spatial resolution extractor, the SPC model is capable of accelerating customization of the plenoptic cameras without the need for expensive measurements.

\section{REFERENCES}

[1] Gabriel Lippmann, "Epreuves reversibles. photographies integrals," Comptes-Rendus Academie des Sciences, vol. 146, pp. 446-451, 1908.

[2] Ren Ng, Digital light field photography, Ph.D. thesis, stanford university, 2006.

[3] Ren Ng, Marc Levoy, Mathieu Brédif, Gene Duval, Mark Horowitz, and Pat Hanrahan, "Light field photography with a hand-held plenoptic camera," Computer Science Technical Report CSTR, vol. 2, 2005.

[4] E.H. Adelson and J.Y.A. Wang, "Single lens stereo with a plenoptic camera," IEEE transactions on pattern analysis and machine intelligence, vol. 14, no. 2, pp. 99-106, 1992. 
[5] Todor Georgiev and Andrew Lumsdaine, "Depth of field in plenoptic cameras," in Proc. Eurographics, 2009, vol. 2009.

[6] A. Lumsdaine and T. Georgiev, "The focused plenoptic camera," in Computational Photography (ICCP), 2009 IEEE International Conference on. IEEE, 2009, pp. 18.

[7] Norbert Lindlein, "Simulation of micro-optical systems including microlens arrays," Journal of Optics A: Pure and Applied Optics, vol. 4, no. 4, pp. S1, 2002.

[8] Christin Langguth, Alexander Oberdörster, Andreas Brückner, Frank Wippermann, and Andreas Bräuer, "Optical performance analysis of plenoptic camera systems," in SPIE Optical Engineering + Applications. International Society for Optics and Photonics, 2014, pp. 91920F-91920F.

[9] Christian Perwass and Lennart Wietzke, "Single lens 3 d-camera with extended depth-of-field," in IS\&T/SPIE Electronic Imaging. International Society for Optics and Photonics, 2012, pp. 829108-829108.

[10] H. Navarro, E. Sánchez-Ortiga, G. Saavedra, A. Llavador, A. Dorado, M. Martínez-Corral, and B. Javidi, "Non-homogeneity of lateral resolution in integral imaging," Journal of Display Technology, vol. 9, no. 1, pp. 37-43, 2013.

[11] Ole Johannsen, Christian Heinze, Bastian Goldluecke, and Christian Perwaß, "On the calibration of focused plenoptic cameras," in Time-of-Flight and Depth Imaging. Sensors, Algorithms, and Applications, pp. 302317. Springer, 2013.

[12] Mitra Damghanian, Roger Olsson, and Mårten Sjöström, "The sampling pattern cube-a representation and evaluation tool for optical capturing systems," in Advanced Concepts for Intelligent Vision Systems. Springer, 2012, pp. 120-131.

[13] Mitra Damghanian, Roger Olsson, Mårten Sjöström, Hector Navarro Fructuoso, and Manuel MartinezCorral, "Investigating the lateral resolution in a plenoptic capturing system using the spc model," in IS\&T/SPIE Electronic Imaging. International Society for Optics and Photonics, 2013, pp. 86600T-86600T.

[14] Mitra Damghanian, Roger Olsson, and Mårten Sjöström, "Performance analysis in Lytro camera: empirical and model based approaches to assess refocusing quality," in Acoustics, Speech and Signal Processing (ICASSP), 2014 IEEE International Conference on. IEEE, 2014, pp. 559-563.
[15] Mitra Damghanian, Roger Olsson, Mårten Sjöström, Arne Erdmann, and Christian Perwass, "Spatial resolution in a multi-focus plenoptic camera," in Image Processing (ICIP), International Conference on. IEEE, 2014, pp. 1932-1936. 